\title{
ON THE EXISTENCE OF PSEUDOHARMONIC MAPS FROM PSEUDOHERMITIAN MANIFOLDS INTO RIEMANNIAN MANIFOLDS WITH NONPOSITIVE SECTIONAL CURVATURE*
}

\author{
SHU-CHENG CHANG ${ }^{\dagger}$ AND TING-HUI CHANG C $^{\ddagger}$
}

\begin{abstract}
In this paper, we first derive a CR Bochner identity for the pseudoharmonic map heat flow on pseudohermitian manifolds. Secondly, we are able to prove existence of the global solution for the pseudoharmonic map heat flow from a closed pseudohermitian manifold into a Riemannian manifold with nonpositive sectional curvature. In particular, we prove the existence theorem of pseudoharmonic maps. This is served as the CR analogue of Eells-Sampson's Theorem for the harmonic map heat flow.
\end{abstract}

Key words. CR Bochner identity, energy density, pseudoharmonic map, pseudoharmonic map heat flow, pseudohermitian manifold, pseudohermitian Ricci tensors, pseudohermitian torsion, subLaplacian, Folland-Stein space.

AMS subject classifications. Primary 32V05, 32V20; Secondary 53C56.

1. Introduction. In the paper of J. Eells and J. H. Sampson ([ES]), they obtained the following remarkable existence theorem of harmonic maps between Riemannian manifolds. Let $\left(M^{n}, h_{\alpha \beta}\right),\left(N^{m}, g_{i j}\right)$ be Riemannian manifolds with $M$ closed and $f: M \rightarrow N$ be a $C^{\infty}$ map. Suppose that the sectional curvature $\widetilde{K}^{N}$ of $N$ is nonpositive, then $f$ can be deformed to a harmonic map. More precisely, they considered the harmonic map heat flow on $M \times[0, T)$ :

$$
\left\{\begin{array}{l}
\frac{\partial u^{k}}{\partial t}-\Delta u^{k}=\widetilde{\Gamma}_{i j}^{k} \frac{\partial u^{i}}{\partial x_{\alpha}} \frac{\partial u^{j}}{\partial x_{\alpha}}, \quad k=1, \cdots, m \\
u(x, 0)=f(x) .
\end{array}\right.
$$

Here $\Delta$ is the Laplace-Beltrami operator and $\widetilde{\Gamma}_{i j}^{k}$ are the Christoffel symbols of $N$. If the sectional curvature $\widetilde{K}^{N}$ of $N$ is nonpositive, then (1.1) admits a unique, smooth solution $u \in C^{\infty}(M \times[0, \infty) ; N)$ which converges to a harmonic map $u_{\infty} \in C^{\infty}(M ; N)$ as $t \rightarrow \infty$ suitably.

In this paper, we will follow the same method to study the global smooth solutions to the pseudoharmonic map heat flow from a closed pseudohermitian manifold $(M, J, \theta)$ to a Riemannian manifold $\left(N, g_{i j}\right)$ and thus obtain the existence theorem of pseudoharmonic maps between a closed pseudohermitian manifold $(M, J, \theta)$ and a Riemannian manifold $\left(N, g_{i j}\right)$.

We first introduce some basic materials in a pseudohermitian $(2 n+1)$-manifold. Let $(M, \xi)$ be a $(2 n+1)$-dimensional, orientable, contact manifold with contact structure $\xi, \operatorname{dim}_{\mathbb{R}} \xi=2 n$. A CR structure compatible with $\xi$ is an endomorphism $J: \xi \rightarrow \xi$ such that $J^{2}=-1$. We also assume that $J$ satisfies the following integrability condition: If $X$ and $Y$ are in $\xi$, then so are $[J X, Y]+[X, J Y]$ and $J([J X, Y]+[X, J Y])=[J X, J Y]-[X, Y]$. A CR structure $J$ can extend to $\mathbb{C} \otimes \xi$

*Received May 23, 2011; accepted for publication February 17, 2012. Research supported in part by the NSC of Taiwan.

${ }^{\dagger}$ Department of Mathematics, National Taiwan University, Taipei 10617, Taiwan, R.O.C. (scchang @math.ntu.edu.tw).

¥Institute of Mathematics, Academia Sinica, Taipei 10617, Taiwan, R.O.C. (thc@math. sinica.edu.tw). 
and decomposes $\mathbb{C} \otimes \xi$ into the direct sum of $T_{1,0}$ and $T_{0,1}$ which are eigenspaces of $J$ with respect to eigenvalues $i$ and $-i$, respectively. A manifold $M$ with a CR structure is called a $\mathrm{CR}$ manifold. A pseudohermitian structure compatible with $\xi$ is a $C R$ structure $J$ compatible with $\xi$ together with a choice of contact form $\theta$. Such a choice determines a unique real vector field $T$ transverse to $\xi$, which is called the characteristic vector field of $\theta$, such that $\theta(T)=1$ and $\mathcal{L}_{T} \theta=0$ or $d \theta(T, \cdot)=0$. Let $\left\{T, Z_{\alpha}, Z_{\bar{\alpha}}\right\}$ be a frame of $T M \otimes \mathbb{C}$, where $Z_{\alpha}$ is any local frame of $T_{1,0}, Z_{\bar{\alpha}}=\overline{Z_{\alpha}} \in T_{0,1}$ and $T$ is the characteristic vector field. Then $\left\{\theta, \theta^{\alpha}, \theta^{\bar{\alpha}}\right\}$, which is the coframe dual to $\left\{T, Z_{\alpha}, Z_{\bar{\alpha}}\right\}$, satisfies

$$
d \theta=i h_{\alpha \bar{\beta}} \theta^{\alpha} \wedge \theta^{\bar{\beta}},
$$

for some positive definite hermitian matrix of functions $\left(h_{\alpha \bar{\beta}}\right)$. Actually we can always choose $Z_{\alpha}$ such that $h_{\alpha \bar{\beta}}=\delta_{\alpha \beta}$; hence, throughout this paper, we assume $h_{\alpha \bar{\beta}}=\delta_{\alpha \beta}$.

The Levi form $\langle,\rangle_{L_{\theta}}$ is the Hermitian form on $T_{1,0}$ defined by

$$
\langle Z, W\rangle_{L_{\theta}}=-i\langle d \theta, Z \wedge \bar{W}\rangle
$$

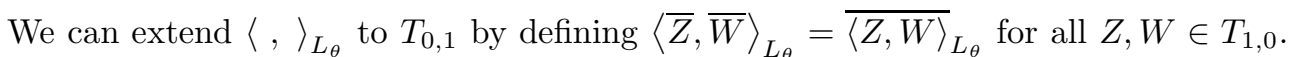
The Levi form induces naturally a Hermitian form on the dual bundle of $T_{1,0}$, denoted by $\langle,\rangle_{L_{\theta}^{*}}$, and hence on all the induced tensor bundles. Integrating the Hermitian form (when acting on sections) over $M$ with respect to the volume form $d \mu=\theta \wedge(d \theta)^{n}$, we get an inner product on the space of sections of each tensor bundle. We denote the inner product by the notation $\langle$,$\rangle . For example$

$$
\langle u, v\rangle=\int_{M} u \bar{v} d \mu
$$

for functions $u$ and $v$.

The pseudohermitian connection of $(J, \theta)$ is the connection $\nabla$ on $T M \otimes \mathbb{C}$ (and extended to tensors) given in terms of a local frame $Z_{\alpha} \in T_{1,0}$ by

$$
\nabla Z_{\alpha}=\theta_{\alpha}^{\beta} \otimes Z_{\beta}, \quad \nabla Z_{\bar{\alpha}}=\theta_{\bar{\alpha}}^{\bar{\beta}} \otimes Z_{\bar{\beta}}, \quad \nabla T=0,
$$

where $\theta_{\alpha}{ }^{\beta}$ are the 1-forms uniquely determined by the following equations:

$$
\begin{aligned}
d \theta^{\beta} & =\theta^{\alpha} \wedge \theta_{\alpha}^{\beta}+\theta \wedge \tau^{\beta}, \\
0 & =\tau_{\alpha} \wedge \theta^{\alpha}, \\
0 & =\theta_{\alpha}{ }^{\beta}+\theta_{\bar{\beta}}^{\bar{\alpha}},
\end{aligned}
$$

We can write (by Cartan lemma) $\tau_{\alpha}=A_{\alpha \gamma} \theta^{\gamma}$ with $A_{\alpha \gamma}=A_{\gamma \alpha}$ the pseudohermitian torsion of $(M, J, \theta)$. The curvature of the Webster-Stanton connection, expressed in terms of the coframe $\left\{\theta=\theta^{0}, \theta^{\alpha}, \theta^{\bar{\alpha}}\right\}$, is

$$
\begin{aligned}
& \Pi_{\beta}{ }^{\alpha}=\overline{\Pi_{\bar{\beta}} \bar{\alpha}}=d \omega_{\beta}{ }^{\alpha}-\omega_{\beta}{ }^{\gamma} \wedge \omega_{\gamma}{ }^{\alpha}, \\
& \Pi_{0}{ }^{\alpha}=\Pi_{\alpha}{ }^{0}=\Pi_{0}{ }^{\bar{\beta}}=\Pi_{\bar{\beta}}{ }^{0}=\Pi_{0}{ }^{0}=0 .
\end{aligned}
$$

Webster showed that $\Pi_{\beta}^{\alpha}$ can be written

$$
\Pi_{\beta}{ }^{\alpha}=R_{\beta}{ }^{\alpha}{ }_{\rho \bar{\sigma}} \theta^{\rho} \wedge \theta^{\bar{\sigma}}+W_{\beta}{ }^{\alpha}{ }_{\rho} \theta^{\rho} \wedge \theta-W^{\alpha}{ }_{\beta \bar{\rho}} \theta^{\bar{\rho}} \wedge \theta+i \theta_{\beta} \wedge \tau^{\alpha}-i \tau_{\beta} \wedge \theta^{\alpha}
$$


where the coefficients satisfy

$$
R_{\beta \bar{\alpha} \rho \bar{\sigma}}=\overline{R_{\alpha \bar{\beta} \sigma \bar{\rho}}}=R_{\bar{\alpha} \beta \bar{\sigma} \rho}=R_{\rho \bar{\alpha} \beta \bar{\sigma}}, \quad W_{\beta \bar{\alpha} \gamma}=W_{\gamma \bar{\alpha} \beta} .
$$

We will denote components of covariant derivatives with indices preceded by comma; thus write $A_{\alpha \beta, \gamma}$. The indices $\{0, \alpha, \bar{\alpha}\}$ indicate derivatives with respect to $\left\{T, Z_{\alpha}, Z_{\bar{\alpha}}\right\}$. For derivatives of a scalar function, we will often omit the comma, for instance, $u_{\alpha}=Z_{\alpha} u, u_{\alpha \bar{\beta}}=Z_{\bar{\beta}} Z_{\alpha} u-\omega_{\alpha}^{\gamma}\left(Z_{\bar{\beta}}\right) Z_{\gamma} u, u_{0}=T u$ for a smooth function $u$

For a real function $u$, the subgradient $\nabla_{b}$ is defined by $\nabla_{b} u \in \xi$ and $\left\langle Z, \nabla_{b} u\right\rangle_{L_{\theta}}=$ $d u(Z)$ for all vector fields $Z$ tangent to contact plane. Locally $\nabla_{b} u=\sum_{\alpha} u_{\bar{\alpha}} Z_{\alpha}+$ $u_{\alpha} Z_{\bar{\alpha}}$. We can use the connection to define the subhessian as the complex linear map

$$
\left(\nabla^{H}\right)^{2} u: T_{1,0} \oplus T_{0,1} \rightarrow T_{1,0} \oplus T_{0,1}
$$

by

$$
\left(\nabla^{H}\right)^{2} u(Z)=\nabla_{Z} \nabla_{b} u
$$

In particular,

$$
\left|\nabla_{b} u\right|^{2}=2 u_{\alpha} u_{\bar{\alpha}}, \quad\left|\nabla_{b}^{2} u\right|^{2}=2\left(u_{\alpha \beta} u_{\bar{\alpha} \bar{\beta}}+u_{\alpha \bar{\beta}} u_{\bar{\alpha} \beta}\right)
$$

Also

$$
\Delta_{b} u=\operatorname{Tr}\left(\left(\nabla^{H}\right)^{2} u\right)=\sum_{\alpha}\left(u_{\alpha \bar{\alpha}}+u_{\bar{\alpha} \alpha}\right) .
$$

The pseudohermitian Ricci tensor and the torsion tensor on $T_{1,0}$ are defined by

$$
\operatorname{Ric}(X, Y)=R_{\alpha \bar{\beta}} X^{\alpha} Y^{\bar{\beta}}
$$

and

$$
\operatorname{Tor}(X, Y)=i \sum_{\alpha, \beta}\left(A_{\bar{\alpha} \bar{\beta}} X^{\bar{\alpha}} Y^{\bar{\beta}}-A_{\alpha \beta} X^{\alpha} Y^{\beta}\right)
$$

where $X=X^{\alpha} Z_{\alpha}, Y=Y^{\beta} Z_{\beta}$.

Denote by Ric and $\widetilde{R}^{N}$ the pseudohermitian Ricci tensor and Riemannian curvature tensor of $\left(M^{2 n+1}, J, \theta\right)$ and $\left(N^{m}, g\right)$, respectively. In a local coordinate chart at $p \in M$, write

$$
\operatorname{Ric}=\left(R_{\alpha \bar{\beta}}\right), \quad \widetilde{R}^{N}=\left(\widetilde{R}_{i j k \ell}\right)
$$

and $\widetilde{K}^{N}$ denotes the sectional curvature of $\left(N^{m}, g\right)$.

Let $\left(M^{2 n+1}, J, \theta\right)$ be a closed pseudohermitian $(2 n+1)$-manifold, $\left(N^{m}, g\right)$ be a Riemannian $m$-manifold and let $\varphi \in C^{2}(M ; N)$. At each point $p \in M$, we may take a local coordinate chart $U_{p} \subset M$ of $p$ and a local coordinate chart $V_{\varphi(p)} \subset N$ of $\varphi(p)$ such that $\varphi\left(U_{p}\right) \subset V_{\varphi(p)}$. We define the energy density $e(\varphi)$ of $\varphi$ at the point $x \in U_{p}$ by

$$
e(\varphi)(x)=\frac{1}{2} h^{\alpha \bar{\beta}}(x) g_{i j}(\varphi(x)) \varphi_{\alpha}^{i} \varphi_{\bar{\beta}}^{j}
$$


Here $h_{\alpha \bar{\beta}}$ is the Levi metric on $\left(M^{2 n+1}, J, \theta\right)$ and we may assume $h_{\alpha \bar{\beta}}=\delta_{\alpha \beta}$. It can be checked that the energy density is intrinsically defined, i.e., independent of the choice of local coordinates.

Now we define the energy $E(\varphi)$ of $\varphi$ by

$$
E(\varphi)=\int_{M} e(\varphi) d \mu
$$

where $d \mu=\theta \wedge(d \theta)^{n}$. In the paper of E. Barletta, S. Dragomir and H. Urakawa ([BDU]), they introduced a notion of the pseudoharmonic map from a pseudohermitian $(2 n+1)$-manifold $\left(M^{2 n+1}, J, \theta\right)$ into a Riemannian $m$-manifold $\left(N^{m}, g\right)$ as following:

Definition 1.1. A $C^{2}$-map $\varphi:\left(M^{2 n+1}, J, \theta\right) \rightarrow\left(N^{m}, g\right)$ is said to be a pseudoharmonic map if it is a critical point of the energy functional $E$.

REMARK 1.1. 1. A $C^{2}$-map $\varphi:(M, J, \theta) \rightarrow(N, g)$ is pseudoharmonic if and only if it satisfies the Euler-Lagrange equations

$$
\Delta_{b} \varphi^{k}+2 \widetilde{\Gamma}_{i j}^{k} \varphi_{\alpha}^{i} \varphi_{\bar{\alpha}}^{j}=0, \quad k=1, \cdots, m,
$$

where $\widetilde{\Gamma}_{i j}^{k}$ are the Christoffel symbols of $\left(N^{m}, g\right)$ ( Lemma 2.2).

2. Pseudoharmonic maps are solutions to a nonlinear subelliptic pde system. It is an Hörmander-type operator. In the paper of J. Jost and C.-J. Xu ([JX]), they studied subelliptic harmonic maps on an open domain in the Euclidean space. In the paper of $R$. Petit ([Pe]), he studied a harmonic map between two pseudohermitian manifolds.

3. Geometrically, they discovered the following phenomenon in $[B D U]:$ Let $K(M)$ be the canonical bundle over $\left(M^{2 n+1}, J, \theta\right)$ and $C(M)=(K(M) \backslash\{0\}) / \mathbf{R}_{+}$be the associated Fefferman manifold which is the circle bundle over $M$. Let $\pi: C(M) \rightarrow M$ be the projection and $h$ be the associated Fefferman metric, a Lorentz metric on $C(M)$ (see [L2] for details). Then $\varphi:\left(M^{2 n+1}, J, \theta\right) \rightarrow(N, g)$ is pseudoharmonic if and only if its vertical lift $\varphi \circ \pi:(C(M), h) \rightarrow(N, g)$ is harmonic. From this point of view, pseudoharmonic maps on a pseudohermitian manifold is closely related to harmonic (wave) maps on the Minkowsky space ([SS]).

The organization of this paper is as follows. In section 2, let's recall two preliminary but important lemmas which play crucial roles in section 3 . The first one gives the CR Bochner identity for a smooth real function on a closed pseudohermitian manifold. The second one gives a necessary and sufficient condition for a $C^{2}$-map to be pseudoharmonic. In section 3 , let $\left(M^{2 n+1}, J, \theta\right)$ be a closed pseudohermitian manifold, $\left(N^{m}, g\right)$ be a Riemannian manifold and we consider the pseudoharmonic map heat flow on $M \times[0, T)$ :

$$
\left\{\begin{array}{l}
\frac{\partial \varphi^{k}}{\partial t}-\Delta_{b} \varphi^{k}=2 \widetilde{\Gamma}_{i j}^{k} \varphi_{\alpha}^{i} \varphi_{\bar{\alpha}}^{j}, \quad k=1, \cdots, m \\
\varphi(x, 0)=f(x), \quad f \in C^{\infty}(M ; N) .
\end{array}\right.
$$

Here $\widetilde{\Gamma}_{i j}^{k}$ are the Christoffel symbols of $N$. We will follow the same method of EellsSampson to show the existence of global smooth solutions to the pseudoharmonic map heat flow (1.2). The main difficulty comes from the CR Bochner formula (2.1) with a 
mixed term $\left\langle J \nabla_{b} u, \nabla_{b} u_{0}\right\rangle_{L_{\theta}}$ involving the covariant derivative of $u$ in the direction of $T$, which is hard to control. However, by adding a certain extra term, we are able to overcome such a difficulty and conclude that (1.2) has a global smooth solution. The method is as follows. We first apply Moser's Harnack inequality to show the uniform upper bound of the energy density of the pseudoharmonic map heat flow and then use the higher order regularity theory of Folland-Stein space $S^{k, p}$ to show the existence of global smooth solution to (1.2).

We recall below what the Folland-Stein space $S^{k, p}$ is. Let $D$ denote a differential operator acting on functions. We say $D$ has weight $m$, denoted $w(D)=m$, if $m$ is the smallest integer such that $D$ can be locally expressed as a polynomial of degree $m$ in vector fields tangent to the contact bundle $\xi$. We define the Folland-Stein space $S^{k, p}$ of functions on $M$ by

$$
S^{k, p}=\left\{f \in L^{p}: D f \in L^{p} \text { whenever } w(D) \leq k\right\} .
$$

We define the $L^{p}$ norm of $\nabla_{b} f, \nabla_{b}^{2} f, \ldots$ to be $\left(\int\left|\nabla_{b} f\right|^{p} \theta \wedge d \theta\right)^{1 / p},\left(\int\left|\nabla_{b}^{2} f\right|^{p} \theta \wedge d \theta\right)^{1 / p}$, $\ldots$, respectively, as usual. So it is natural to define the $S^{k, p}$ norm of $f \in S^{k, p}$ as follows:

$$
\|f\|_{S^{k, p}} \equiv\left(\sum_{0 \leq j \leq k}\left\|\nabla_{b}^{j} f\right\|_{L^{p}}^{p}\right)^{1 / p} .
$$

The function space $S^{k, p}$ with the above norm is a Banach space for $k \geq 0,1<p<\infty$. There are also embedding theorems of Sobolev type. The reader can make reference to $[\mathrm{CCC}],[\mathrm{F}]$ and $[\mathrm{FS}]$ for more details of these spaces.

In order to apply Moser's Harnack inequality to show that the energy density of (1.2) is uniformly bounded, we have to consider an extra energy density

$$
e_{0}(\varphi):=g_{i j} \varphi_{0}^{i} \varphi_{0}^{j}
$$

and then estimate the total energy density

$$
\widehat{e}(\varphi)=2 e(\varphi)+e_{0}(\varphi)
$$

In fact, we will show that

THEOREM 1.1. Let $\left(M^{2 n+1}, J, \theta\right)$ be a closed pseudohermitian manifold, $\left(N^{m}, g\right)$ be a Riemannian manifold with nonpositive sectional curvature $\widetilde{K}^{N}$. Let $\varphi \in C^{\infty}(M \times$ $[0, T) ; N)$ be a solution of (1.2). If

$$
\left[\Delta_{b}, T\right]=0
$$

then it holds

$$
\left(\frac{\partial}{\partial t}-\Delta_{b}\right) \widehat{e}(\varphi) \leq C \widehat{e}(\varphi)
$$

Here $C$ is a positive constant depends on the pseudohermitian Ricci tensor and torsion of $(M, J, \theta)$.

In the paper of C. R. Graham and J. M. Lee ([GL]), they introduced the purely holomorphic second-order operator

$$
Q u=2 i\left(A_{\bar{\alpha} \bar{\beta}} u_{\alpha}\right)_{, \beta}
$$


and showed that

$$
\left[\Delta_{b}, T\right]=2 \operatorname{Im}(Q)
$$

In particular, if the pseudohermitian torsion $A_{\alpha \beta}$ of $M$ is vanishing, the sublaplacian operator $\Delta_{b}$ commutes with the characteristic vector field $T$ and we have

COROLlary 1.2. Let $\left(M^{2 n+1}, J, \theta\right)$ be a closed pseudohermitian manifold with vanishing pseudohermitian torsion and $\left(N^{m}, g\right)$ be a Riemannian manifold with nonpositive sectional curvature $\widetilde{K}^{N}$. Let $\varphi \in C^{\infty}(M \times[0, T) ; N)$ be a solution of (1.2), then it holds

$$
\left(\frac{\partial}{\partial t}-\Delta_{b}\right) \widehat{e}(\varphi) \leq C \widehat{e}(\varphi)
$$

Here $C$ is a constant depends on the pseudohermitian Ricci tensor of $(M, J, \theta)$.

Finally, with the help of Theorem 1.1, we are able to prove our main theorem, which is a CR analogue result of Eells-Sampson theorem that any smooth map, from a closed pseudohermitian manifold $\left(M^{2 n+1}, J, \theta\right)$ to a Riemannian manifold $\left(N^{m}, g\right)$ with nonpositive sectional curvature $\widetilde{K}^{N}$, can be deformed to a pseudoharmonic map. That is, we prove the following existence theorem of pseudoharmonic maps from a closed pseudohermitian manifold $\left(M^{2 n+1}, J, \theta\right)$ into a Riemannian manifold $\left(N^{m}, g\right)$.

THEOREM 1.3. Let $\left(M^{2 n+1}, J, \theta\right)$ be a closed pseudohermitian manifold, $\left(N^{m}, g\right)$ be a Riemannian manifold with nonpositive sectional curvature $\widetilde{K}^{N}$. Assume that

$$
\left[\Delta_{b}, T\right]=0
$$

Then for any $f \in C^{\infty}(M ; N)$, the pseudoharmonic map heat flow (1.2) admits a unique, smooth solution $\varphi \in C^{\infty}(M \times[0, \infty) ; N)$ which subconverges to a pseudoharmonic $\operatorname{map} \varphi_{\infty} \in C^{\infty}(M ; N)$ as $t \rightarrow \infty$.

Corollary 1.4. Let $\left(M^{2 n+1}, J, \theta\right)$ be a closed pseudohermitian manifold with vanishing pseudohermitian torsion and $\left(N^{m}, g\right)$ be a Riemannian manifold with nonpositive sectional curvature $\widetilde{K}^{N}$. Then for any $f \in C^{\infty}(M ; N)$, the pseudoharmonic map heat flow (1.2) admits a unique, smooth solution $\varphi \in C^{\infty}(M \times[0, \infty) ; N)$ which subconverges to a pseudoharmonic map $\varphi_{\infty} \in C^{\infty}(M ; N)$ as $t \rightarrow \infty$.

2. Euler-Lagrange equation and CR Bochner formula. In this section, we first derive the CR version of Bochner formula for a real smooth function on a closed pseudohermitian manifold $\left(M^{2 n+1}, J, \theta\right)$.

Lemma 2.1. Let $\left(M^{2 n+1}, J, \theta\right)$ be a closed pseudohermitian manifold. For a real smooth function $u$ on $(M, J, \theta)$,

$$
\begin{aligned}
\frac{1}{2} \Delta_{b}\left|\nabla_{b} u\right|^{2}= & \left|\left(\nabla^{H}\right)^{2} u\right|^{2}+\left\langle\nabla_{b} u, \nabla_{b} \Delta_{b} u\right\rangle_{L_{\theta}} \\
& +[2 R i c-(n-2) \text { Tor }]\left(\left(\nabla_{b} u\right)_{\mathbf{C}},\left(\nabla_{b} u\right)_{\mathbf{C}}\right)+2\left\langle J \nabla_{b} u, \nabla_{b} u_{0}\right\rangle_{L_{\theta}} .
\end{aligned}
$$

Here $\left(\nabla_{b} u\right)_{\mathbf{C}}=u_{\bar{\alpha}} Z_{\alpha}$ is the corresponding complex $(1,0)$-vector field of $\nabla_{b} u$ and $d_{b} u=u_{\alpha} \theta^{\alpha}+u_{\bar{\alpha}} \theta^{\bar{\alpha}}$. 
Proof. First from [GL], we have for a real smooth function $u$

$$
\begin{aligned}
\frac{1}{2} \Delta_{b}\left|\nabla_{b} u\right|^{2}= & \left|\nabla_{b}^{2} u\right|^{2}+\left\langle\nabla_{b} u, \nabla_{b} \Delta_{b} u\right\rangle_{L_{\theta}} \\
& +[2 \text { Ric }-n \text { Tor }]\left(\left(\nabla_{b} u\right)_{C},\left(\nabla_{b} u\right)_{C}\right)-2 i \sum_{\alpha=1}^{n}\left(u_{\alpha} u_{\bar{\alpha} 0}-u_{\bar{\alpha}} u_{\alpha 0}\right) .
\end{aligned}
$$

Next from Lemma 2.2 of [CC1], one has that

$$
\begin{aligned}
i \sum_{\alpha}\left(u_{\alpha} u_{\bar{\alpha} 0}-u_{\bar{\alpha}} u_{\alpha 0}\right) & =i \sum_{\alpha}\left(u_{\alpha} u_{0 \bar{\alpha}}-u_{\bar{\alpha}} u_{0 \alpha}\right)-\operatorname{Tor}\left(\left(\nabla_{b} u\right)_{C},\left(\nabla_{b} u\right)_{C}\right) \\
& =-\left\langle J \nabla_{b} u, \nabla_{b} u_{0}\right\rangle_{L_{\theta}}-\operatorname{Tor}\left(\left(\nabla_{b} u\right)_{C},\left(\nabla_{b} u\right)_{C}\right) .
\end{aligned}
$$

Then Lemma 2.1 follows from (2.2) and (2.3).

The following lemma gives a necessary and sufficient condition for a map $\varphi \in$ $C^{2}(M ; N)$ to be pseudoharmonic. We refer to [BDU] for another derivation.

LEMMA 2.2. Let $\left(M^{2 n+1}, J, \theta\right)$ be a closed pseudohermitian manifold and $\left(N^{m}, g\right)$ be a Riemannian manifold. A $C^{2}$-map $\varphi:(M, J, \theta) \rightarrow(N, g)$ is pseudoharmonic if and only if it satisfies the Euler-Lagrange equations

$$
\Delta_{b} \varphi^{k}+2 \widetilde{\Gamma}_{i j}^{k} \varphi_{\alpha}^{i} \varphi_{\bar{\alpha}}^{j}=0, \quad k=1, \cdots, m,
$$

where $\widetilde{\Gamma}_{i j}^{k}$ are the Christoffel symbols of $\left(N^{m}, g\right)$.

Proof. Let $\varphi_{t},-\varepsilon<t<\varepsilon$, be a smooth variation of $\varphi$ so that

$$
\varphi_{0}=\varphi, \quad \text { and }\left.\quad \frac{d \varphi_{t}}{d t}\right|_{t=0}=V \in \Gamma\left(\varphi^{-1} T N\right)
$$

$\varphi_{t}$ may be viewed as a map from $(-\varepsilon, \varepsilon) \times M$ into $N$. By direct computations one has

$$
\begin{aligned}
\frac{d}{d t} E\left(\varphi_{t}\right) & =\frac{1}{2} \frac{d}{d t} \int_{M} g_{i j}\left(\varphi_{t}\right) \varphi_{t \alpha}^{i} \varphi_{t \bar{\alpha}}^{j} d \mu \\
& =-\frac{1}{2} \int_{M} g_{k \ell}\left[\Delta_{b} \varphi_{t}^{k}+2 \widetilde{\Gamma}_{i j}^{k} \varphi_{t \alpha}^{i} \varphi_{t \bar{\alpha}}^{j}\right] \frac{d \varphi_{t}^{\ell}}{d t} d \mu \\
& =-\frac{1}{2} \int_{M}\left\langle\frac{d \varphi_{t}}{d t}, \tau\left(\varphi_{t}\right)\right\rangle_{N} d \mu .
\end{aligned}
$$

Thus, the first variational formula is given by

$$
\left.\frac{d}{d t} E\left(\varphi_{t}\right)\right|_{t=0}=-\frac{1}{2} \int_{M}\langle V, \tau(\varphi)\rangle_{N} d \mu,
$$

where $\tau(\varphi)$ is so-called the tension field of $\varphi$, which is defined by

$$
\tau(\varphi)=\sum_{k=1}^{m}\left[\Delta_{b} \varphi^{k}+2 \widetilde{\Gamma}_{i j}^{k} \varphi_{\alpha}^{i} \varphi_{\bar{\alpha}}^{j}\right] \frac{\partial}{\partial y_{k}} .
$$

Therefore $\varphi \in C^{2}(M ; N)$ is a critical point of the energy functional $E$ if and only if its tension field $\tau(\varphi)$ vanishes identically. That is, $\varphi$ is pseudoharmonic if and only if it satisfies the Euler-Lagrange equations (2.4). 
3. Existence of global smooth solutions under $\widetilde{K}^{N} \leq 0$. In this section, we will study the pseudoharmonic map heat flow (1.2) and follow the same method of Eells-Sampson to show the existence of global smooth solutions under $\widetilde{K}^{N} \leq 0$.

We first need the following lemma, which states that the energy $E(\varphi(t))$ for $(1.2)$ is monotonically decreasing.

Lemma 3.1. For any $0<T \leq \infty$, if $\varphi \in C^{\infty}(M \times[0, T) ; N)$ solves (1.2), then

$$
E(\varphi(t))+\frac{1}{2} \int_{0}^{t} \int_{M}\left|\partial_{s} \varphi\right|^{2} d \mu d s=E(f), \quad \forall t \in[0, T) .
$$

Proof. By integration by parts, one has

$$
\begin{aligned}
& \frac{d}{d s} E(\varphi(s))=\frac{1}{2} \int_{M} \frac{\partial}{\partial s}\left(g_{i j} \varphi_{\beta}^{i} \varphi_{\bar{\beta}}^{j}\right) d \mu \\
= & \frac{1}{2} \int_{M} g_{i j, k} \varphi_{\beta}^{i} \varphi_{\frac{j}{\beta}} \frac{\partial \varphi^{k}}{\partial s} d \mu-\frac{1}{2} \int_{M} g_{i j} \frac{\partial \varphi^{i}}{\partial s} \varphi_{\beta \bar{\beta}}^{j} d \mu-\frac{1}{2} \int_{M} g_{i j, k} \varphi_{\beta}^{k} \varphi_{\frac{j}{\beta}} \frac{\partial \varphi^{i}}{\partial s} d \mu \\
& \quad-\frac{1}{2} \int_{M} g_{i j} \frac{\partial \varphi^{j}}{\partial s} \varphi_{\bar{\beta}}^{i} d \mu-\frac{1}{2} \int_{M} g_{i j, k} \varphi_{\beta}^{i} \varphi_{\frac{k}{\beta}} \frac{\partial \varphi^{j}}{\partial s} d \mu \\
= & \frac{1}{2}-\int_{M} g_{i j} \frac{\partial \varphi^{i}}{\partial s} \Delta_{b} \varphi^{j} d \mu-\frac{1}{2} \int_{M}\left(g_{k j, i}+g_{i k, j}-g_{i j, k}\right) \varphi_{\beta}^{i} \varphi_{\frac{j}{\beta}} \frac{\partial \varphi^{k}}{\partial s} d \mu \\
= & -\frac{1}{2} \int_{M} g_{i j} \frac{\partial \varphi^{i}}{\partial s}\left[\Delta_{b} \varphi^{j}+2 \widetilde{\Gamma}_{p q}^{j} \varphi_{\beta}^{p} \varphi_{\frac{q}{\beta}}\right] d \mu \\
= & -\frac{1}{2} \int_{M} g_{i j} \frac{\partial \varphi^{i}}{\partial s} \frac{\partial \varphi^{j}}{\partial s} d \mu .
\end{aligned}
$$

That is,

$$
\frac{d}{d s} E(\varphi(s))=-\frac{1}{2} \int_{M}\left|\partial_{s} \varphi\right|^{2} d \mu
$$

Integrating the above equality over $[0, t]$ gives $(3.1)$.

We next derive the $\mathrm{CR}$ version of Bochner identity for the pseudoharmonic map heat flow (1.2).

LEMmA 3.2. Let $\left(M^{2 n+1}, J, \theta\right)$ be a closed pseudohermitian manifold, $\left(N^{m}, g\right)$ be a Riemannian manifold and let $\varphi \in C^{\infty}(M \times[0, T) ; N)$ be a solution to the pseudoharmonic map heat flow (1.2). If $\left[\Delta_{b}, T\right]=0$, then there holds

$$
\begin{aligned}
& \left(\frac{\partial}{\partial t}-\Delta_{b}\right) \widehat{e}(\varphi) \\
= & -\sum_{k=1}^{m}\left[\begin{array}{c}
\left.\left|\nabla_{b}^{2} \varphi^{k}\right|^{2}+(2 R i c-(n-2) T o r)\left(\left(\nabla_{b} \varphi^{k}\right)_{C},\left(\nabla_{b} \varphi^{k}\right)_{C}\right)\right] \\
+2\left\langle J \nabla_{b} \varphi^{k}, \nabla_{b} \varphi_{0}^{k}\right\rangle_{L_{\theta}}+2\left|\nabla_{b} \varphi_{0}^{k}\right|^{2}
\end{array}\right] \\
& +\sum_{i j, k, \ell=1}^{m} \sum_{\alpha, \beta=1}^{n}\left[2 \widetilde{R}_{i j k \ell} \varphi_{\alpha}^{i} \varphi_{\beta}^{j} \varphi \frac{k}{\alpha} \varphi \frac{\ell}{\beta}+2 \widetilde{R}_{i j k \ell} \varphi_{\alpha}^{i} \varphi \frac{j}{\beta} \varphi \frac{k}{\alpha} \varphi_{\beta}^{\ell}\right] \\
& +4 \sum_{i, j, k, \ell=1}^{m} \sum_{\alpha=1}^{n} \widetilde{R}_{i j k \ell} \varphi_{\alpha}^{i} \varphi_{0}^{j} \varphi \frac{k}{\alpha} \varphi_{0}^{\ell} .
\end{aligned}
$$


Proof. Recall that $\widehat{e}(\varphi)=2 e(\varphi)+e_{0}(\varphi)$ with

$$
e_{0}(\varphi):=g_{i j} \varphi_{0}^{i} \varphi_{0}^{j}
$$

Since $e(\varphi)$ and $\widehat{e}(\varphi)$ are independent of the choice of local coordinates, for each point $(p, t)$ one may choose a normal coordinate chart $U$ at $(p, t)$ and a normal coordinate chart $V$ at $\varphi(p, t)$ such that $\varphi(U) \subset V$ and then fulfill the following computations at the point $(p, t)$.

(a) We first compute $\left(\frac{\partial}{\partial t}-\Delta_{b}\right)(2 e(\varphi))$.

$$
\begin{aligned}
& \frac{\partial}{\partial t}(2 e(\varphi)) \\
& =\frac{\partial}{\partial t}\left(\sum_{i, j=1}^{m} \sum_{\beta=1}^{n} g_{i j} \varphi_{\beta}^{i} \varphi_{\bar{\beta}}^{j}\right) \\
& =\sum_{k=1}^{m} \sum_{\beta=1}^{n}\left\{\varphi_{\beta}^{k}\left(\frac{\partial \varphi^{k}}{\partial t}\right)_{\bar{\beta}}+\varphi \frac{k}{\beta}\left(\frac{\partial \varphi^{k}}{\partial t}\right)_{\beta}\right\} \\
& =\sum_{k=1}^{m} \sum_{\beta=1}^{n}\left[\varphi_{\beta}^{k}\left(\Delta_{b} \varphi^{k}+2 \sum_{\alpha=1}^{n} \widetilde{\Gamma}_{i j}^{k} \varphi_{\alpha}^{i} \varphi_{\bar{\alpha}}^{j}\right)_{\bar{\beta}}+\varphi \frac{k}{\beta}\left(\Delta_{b} \varphi^{k}+2 \sum_{\alpha=1}^{n} \widetilde{\Gamma}_{i j}^{k} \varphi_{\alpha}^{i} \varphi_{\bar{\alpha}}^{j}\right)_{\beta}\right] \\
& =\sum_{k=1}^{m}\left\langle\nabla_{b} \varphi^{k}, \nabla_{b} \Delta_{b} \varphi^{k}\right\rangle_{L_{\theta}}+2 \sum_{i, j, k, \ell=1}^{m} \sum_{\alpha, \beta=1}^{n}\left[\widetilde{\Gamma}_{i j, \ell}^{k} \varphi_{\alpha}^{i} \varphi_{\bar{\alpha}}^{j} \varphi_{\beta}^{k} \varphi_{\beta}^{\ell}+\widetilde{\Gamma}_{i j, \ell}^{k} \varphi_{\alpha}^{i} \varphi_{\bar{\alpha}}^{j} \varphi_{\beta}^{k} \varphi_{\beta}^{\ell}\right] .
\end{aligned}
$$

From the CR Bochner formula (Lemma 2.1), one has

$$
\begin{aligned}
\Delta_{b}(2 e(\varphi))= & \Delta_{b}\left(\sum_{i, j=1}^{m} g_{i j} \varphi_{\alpha}^{i} \varphi_{\bar{\alpha}}^{j}\right) \\
= & \frac{1}{2} \sum_{k=1}^{m} \Delta_{b}\left|\nabla_{b} \varphi^{k}\right|^{2}+\sum_{i, j=1}^{m} \varphi_{\alpha}^{i} \varphi_{\bar{\alpha}}^{j} \Delta_{b}\left(g_{i j}\right) \\
= & \sum_{k=1}^{m}\left[\left|\nabla_{b}^{2} \varphi^{k}\right|^{2}+\left\langle\nabla_{b} \varphi^{k}, \nabla_{b} \Delta_{b} \varphi^{k}\right\rangle_{L_{\theta}}+2\left\langle J \nabla_{b} \varphi^{k}, \nabla_{b} \varphi_{0}^{k}\right\rangle_{L_{\theta}}\right. \\
& \left.\quad+(2 R i c-(n-2) \operatorname{Tor})\left(\left(\nabla_{b} \varphi^{k}\right)_{C},\left(\nabla_{b} \varphi^{k}\right)_{C}\right)\right] \\
& +\sum_{i, j=1}^{m} \sum_{\alpha=1}^{n} \varphi_{\alpha}^{i} \varphi_{\bar{\alpha}}^{j} \Delta_{b}\left(g_{i j}\right) .
\end{aligned}
$$

Thus

$$
\begin{aligned}
& \left(\frac{\partial}{\partial t}-\Delta_{b}\right)(2 e(\varphi)) \\
= & -\sum_{k=1}^{m}\left[\begin{array}{c}
\left|\nabla_{b}^{2} \varphi^{k}\right|^{2}+2\left\langle J \nabla_{b} \varphi^{k}, \nabla_{b} \varphi_{0}^{k}\right\rangle_{L_{\theta}} \\
+(2 R i c-(n-2) T o r)\left(\left(\nabla_{b} \varphi^{k}\right)_{C},\left(\nabla_{b} \varphi^{k}\right)_{C}\right)
\end{array}\right] \\
& +2 \sum_{i, j, k, \ell=1}^{m} \sum_{\alpha, \beta=1}^{n}\left[\widetilde{\Gamma}_{i j, \ell}^{k} \varphi_{\alpha}^{i} \varphi_{\bar{\alpha}}^{j} \varphi_{\beta}^{k} \varphi \frac{\ell}{\beta}+\widetilde{\Gamma}_{i j, \ell}^{k} \varphi_{\alpha}^{i} \varphi_{\frac{\alpha}{\alpha}}^{j} \varphi_{\beta}^{k} \varphi_{\beta}^{\ell}\right]-\sum_{i, j=1}^{m} \sum_{\alpha=1}^{n} \varphi_{\alpha}^{i} \varphi_{\bar{\alpha}}^{j} \Delta_{b}\left(g_{i j}\right) .
\end{aligned}
$$


Furthermore at the point $(p, t)$,

$$
\begin{aligned}
& 2 \sum_{i, j, k, \ell=1}^{m} \sum_{\alpha, \beta=1}^{n}\left[\widetilde{\Gamma}_{i j, \ell}^{k} \varphi_{\alpha}^{i} \varphi_{\alpha}^{j} \varphi_{\beta}^{k} \varphi_{\beta}^{\ell}+\widetilde{\Gamma}_{i j, \ell}^{k} \varphi_{\alpha}^{i} \varphi_{\alpha}^{j} \varphi_{\beta}^{\frac{k}{\beta}} \varphi_{\beta}^{\ell}\right]-\sum_{i, j=1}^{m} \sum_{\alpha=1}^{n} \varphi_{\alpha}^{i} \varphi_{\bar{\alpha}}^{j} \Delta_{b}\left(g_{i j}\right) \\
& =\sum_{i, j, k, \ell=1}^{m} \sum_{\alpha, \beta=1}^{n}\left[2 \widetilde{\Gamma}_{i j, \ell}^{k} \varphi_{\alpha}^{i} \varphi_{\alpha}^{j} \varphi_{\beta}^{k} \varphi_{\frac{\ell}{\beta}}+2 \widetilde{\Gamma}_{i j, \ell}^{k} \varphi_{\alpha}^{i} \varphi_{\alpha}^{j} \varphi_{\frac{k}{\beta}}^{k} \varphi_{\beta}^{\ell}-2 g_{i j, k \ell} \varphi_{\alpha}^{i} \varphi_{\frac{j}{\alpha}} \varphi_{\beta}^{k} \varphi_{\frac{\ell}{\beta}}\right] \\
& =\sum_{i, j, k, \ell=1}^{m} \sum_{\alpha, \beta=1}^{n}\left[\left(g_{k j, i \ell}+g_{i k, j \ell}-g_{i j, k \ell}\right) \varphi_{\alpha}^{i} \varphi_{\alpha}^{j} \varphi_{\beta}^{k} \varphi \frac{\ell}{\beta}\right. \\
& \left.-\left(g_{i k, j \ell}+g_{k j, i \ell}-g_{i j, k \ell}\right) \varphi_{\alpha}^{i} \varphi_{\alpha}^{j} \varphi_{\frac{k}{\beta}}^{\ell} \varphi_{\beta}^{\ell}-2 g_{i j, k \ell} \varphi_{\alpha}^{i} \varphi_{\alpha}^{j} \varphi_{\beta}^{k} \varphi \frac{\ell}{\beta}\right] \\
& =\sum_{i, j, k, \ell=1}^{m} \sum_{\alpha, \beta=1}^{n} g_{i j, k \ell}\left[\varphi_{\alpha}^{i} \varphi_{\beta}^{j} \varphi \frac{k}{\alpha} \varphi \frac{\ell}{\beta}+\varphi_{\alpha}^{i} \varphi_{\bar{\beta}}^{j} \varphi_{\alpha}^{k} \varphi_{\beta}^{\ell}\right. \\
& \left.+\varphi_{\alpha}^{i} \varphi_{\bar{\beta}}^{j} \varphi_{\alpha}^{k} \varphi_{\beta}^{\ell}+\varphi_{\alpha}^{i} \varphi_{\beta}^{j} \varphi_{\alpha}^{k} \varphi \frac{\ell}{\beta}-4 \varphi_{\alpha}^{i} \varphi_{\frac{j}{\alpha}} \varphi_{\beta}^{k} \varphi \frac{\ell}{\beta}\right] .
\end{aligned}
$$

On the other hand, we have

$$
\begin{aligned}
& \sum_{i, j, k, \ell=1}^{m} \sum_{\alpha, \beta=1}^{n}\left[2 \widetilde{R}_{i j k \ell} \varphi_{\alpha}^{i} \varphi_{\beta}^{j} \varphi \frac{k}{\alpha} \varphi \frac{\ell}{\beta}+2 \widetilde{R}_{i j k \ell} \varphi_{\alpha}^{i} \varphi_{\frac{j}{\beta}}^{j} \varphi_{\alpha}^{\frac{k}{\alpha}} \varphi_{\beta}^{\ell}\right] \\
&= \sum_{i, j, k, \ell=1}^{m} \sum_{\alpha, \beta=1}^{n}\left[2\left(\widetilde{\Gamma}_{j \ell, k}^{i}-\widetilde{\Gamma}_{j k, \ell}^{i}\right) \varphi_{\alpha}^{i} \varphi_{\beta}^{j} \varphi \frac{k}{\alpha} \varphi_{\frac{\ell}{\beta}}+2\left(\widetilde{\Gamma}_{j \ell, k}^{i}-\widetilde{\Gamma}_{j k, \ell}^{i}\right) \varphi_{\alpha}^{i} \varphi \frac{j}{\beta} \varphi_{\frac{k}{\alpha}} \varphi_{\beta}^{\ell}\right] \\
&= \sum_{i, j, k, \ell=1}^{m} \sum_{\alpha, \beta=1}^{n}\left[\left(g_{i \ell, j k}+g_{j i, \ell k}-g_{j \ell, i k}\right)-\left(g_{i k, j \ell}+g_{j i, k \ell}-g_{j k, i \ell}\right)\right] \varphi_{\alpha}^{i} \varphi_{\beta}^{j} \varphi \frac{k}{\alpha} \varphi_{\frac{\ell}{\beta}} \\
&+\sum_{i, j, k, \ell=1}^{m} \sum_{\alpha, \beta=1}^{n}\left[\left(g_{i \ell, j k}+g_{j i, \ell k}-g_{j \ell, i k}\right)-\left(g_{i k, j \ell}+g_{j i, k \ell}-g_{j k, i \ell}\right)\right] \varphi_{\alpha}^{i} \varphi \frac{j}{\beta} \varphi_{\frac{k}{\alpha}} \varphi_{\beta}^{\ell} \\
&= \sum_{i, j, k, \ell=1}^{m} \sum_{\alpha, \beta=1}^{n} g_{i j, k \ell}\left[\varphi_{\alpha}^{i} \varphi_{\beta}^{j} \varphi_{\frac{k}{\alpha}} \varphi \frac{\ell}{\beta}+\varphi_{\frac{i}{\alpha}} \varphi_{\frac{j}{\beta}} \varphi_{\alpha}^{k} \varphi_{\beta}^{\ell}\right. \\
&\left.+\varphi_{\alpha}^{i} \varphi_{\frac{j}{\beta}}^{j} \varphi \frac{k}{\alpha} \varphi_{\beta}^{\ell}+\varphi \frac{i}{\alpha} \varphi_{\beta}^{j} \varphi_{\alpha}^{k} \varphi \frac{\ell}{\beta}-4 \varphi_{\alpha}^{i} \varphi_{\frac{\alpha}{\alpha}}^{j} \varphi_{\beta}^{k} \varphi \frac{\ell}{\beta}\right] .
\end{aligned}
$$

Therefore, equation (3.2) gives

$$
\begin{aligned}
& \left(\frac{\partial}{\partial t}-\Delta_{b}\right)(2 e(\varphi)) \\
= & -\sum_{k=1}^{m}\left[\begin{array}{c}
\left|\nabla_{b}^{2} \varphi^{k}\right|^{2}+2\left\langle J \nabla_{b} \varphi^{k}, \nabla_{b} \varphi_{0}^{k}\right\rangle_{L_{\theta}} \\
+(2 R i c-(n-2) T o r)\left(\left(\nabla_{b} \varphi^{k}\right)_{C},\left(\nabla_{b} \varphi^{k}\right)_{C}\right)
\end{array}\right] \\
& +\sum_{i, j, k, \ell=1}^{m} \sum_{\alpha, \beta=1}^{n}\left[2 \widetilde{R}_{i j k \ell} \varphi_{\alpha}^{i} \varphi_{\beta}^{j} \varphi \frac{k}{\alpha} \varphi \frac{\ell}{\beta}+2 \widetilde{R}_{i j k \ell} \varphi_{\alpha}^{i} \varphi_{\frac{j}{\beta}} \varphi_{\alpha}^{k} \varphi_{\beta}^{\ell}\right] .
\end{aligned}
$$

(b) We next compute $\left(\frac{\partial}{\partial t}-\Delta_{b}\right) e_{0}(\varphi)$. Again, we have at the point $(p, t)$, that

$$
\frac{\partial}{\partial t}\left(e_{0}(\varphi)\right)=\frac{\partial}{\partial t}\left(\sum_{i, j=1}^{m} g_{i j} \varphi_{0}^{i} \varphi_{0}^{j}\right)=2 \sum_{k=1}^{m} \varphi_{0}^{k}\left(\frac{\partial \varphi^{k}}{\partial t}\right)_{0} .
$$


We also compute

$$
\Delta_{b}\left(e_{0}(\varphi)\right)=\Delta_{b}\left(\sum_{i, j=1}^{m} g_{i j} \varphi_{0}^{i} \varphi_{0}^{j}\right)=\sum_{k=1}^{m}\left[2 \varphi_{0}^{k} \Delta_{b} \varphi_{0}^{k}+2\left|\nabla_{b} \varphi_{0}^{k}\right|^{2}\right]+\sum_{i, j=1}^{m} \varphi_{0}^{i} \varphi_{0}^{j} \Delta_{b}\left(g_{i j}\right)
$$

Thus, under the assumption that $\left[\Delta_{b}, T\right]=0$ we have

$$
\begin{aligned}
& \left(\frac{\partial}{\partial t}-\Delta_{b}\right)\left(e_{0}(\varphi)\right) \\
= & \sum_{k=1}^{m}\left[2\left(\left(\frac{\partial}{\partial t}-\Delta_{b}\right) \varphi^{k}\right)_{0} \varphi_{0}^{k}-2\left|\nabla_{b} \varphi_{0}^{k}\right|^{2}\right]-\sum_{i, j=1}^{m} \varphi_{0}^{i} \varphi_{0}^{j} \Delta_{b}\left(g_{i j}\right) \\
= & 4 \sum_{i, j, k, \ell=1}^{m} \sum_{\alpha=1}^{n} \widetilde{\Gamma}_{i j, \ell}^{k} \varphi_{\alpha}^{i} \varphi_{\frac{\alpha}{\alpha}}^{j} \varphi_{0}^{k} \varphi_{0}^{\ell}-\sum_{i, j=1}^{m} \varphi_{0}^{i} \varphi_{0}^{j} \Delta_{b}\left(g_{i j}\right)-2 \sum_{k=1}^{m}\left|\nabla_{b} \varphi_{0}^{k}\right|^{2} .
\end{aligned}
$$

As what we computed in part (a), it is easy to see that

$$
4 \sum_{i, j, k, \ell=1}^{m} \sum_{\alpha=1}^{n} \widetilde{\Gamma}_{i j, \ell}^{k} \varphi_{\alpha}^{i} \varphi_{\bar{\alpha}}^{j} \varphi_{0}^{k} \varphi_{0}^{\ell}-\sum_{i, j=1}^{m} \varphi_{0}^{i} \varphi_{0}^{j} \Delta_{b}\left(g_{i j}\right)=4 \sum_{i, j, k, \ell=1}^{m} \sum_{\alpha=1}^{n} \widetilde{R}_{i j k \ell} \varphi_{\alpha}^{i} \varphi_{0}^{j} \varphi_{\bar{\alpha}}^{k} \varphi_{0}^{\ell}
$$

and so

$$
\left(\frac{\partial}{\partial t}-\Delta_{b}\right)\left(e_{0}(\varphi)\right)=4 \sum_{i, j, k, \ell=1}^{m} \sum_{\alpha=1}^{n} \widetilde{R}_{i j k \ell} \varphi_{\alpha}^{i} \varphi_{0}^{j} \varphi \frac{k}{\alpha} \varphi_{0}^{\ell}-2 \sum_{k=1}^{m}\left|\nabla_{b} \varphi_{0}^{k}\right|^{2}
$$

Therefore, Lemma 3.2 follows from (3.3) and (3.5). $\square$

With the CR Bochner identity for (1.2), we can now prove Theorem 1.1 and then obtain the uniform upper bound of $e(\varphi)$.

Proof. By Lemma 3.2 and using the Cauchy inequality, one has

$$
\begin{aligned}
& \left(\frac{\partial}{\partial t}-\Delta_{b}\right) \widehat{e}(\varphi) \\
\leq & -\sum_{k=1}^{m}\left[\left|\nabla_{b}^{2} \varphi^{k}\right|^{2}+(2 \text { Ric }-(n-2) \operatorname{Tor})\left(\left(\nabla_{b} \varphi^{k}\right)_{C},\left(\nabla_{b} \varphi^{k}\right)_{C}\right)\right] \\
& +\sum_{k=1}^{m}\left[(\varepsilon-2)\left|\nabla_{b} \varphi_{0}^{k}\right|^{2}+\frac{1}{\varepsilon}\left|\nabla_{b} \varphi^{k}\right|^{2}\right]+2 \widetilde{R}+4 \widetilde{R}^{0}
\end{aligned}
$$

for some positive constant $\varepsilon$. Here

$$
\widetilde{R}=\sum_{i j, k, \ell=1}^{m} \sum_{\alpha, \beta=1}^{n}\left[\widetilde{R}_{i j k \ell} \varphi_{\alpha}^{i} \varphi_{\beta}^{j} \varphi \frac{k}{\alpha} \varphi \frac{\ell}{\beta}+\widetilde{R}_{i j k \ell} \varphi_{\alpha}^{i} \varphi_{\frac{j}{\beta}} \varphi_{\frac{k}{\alpha}} \varphi_{\beta}^{\ell}\right]
$$

and

$$
\widetilde{R}^{0}=\sum_{i, j, k, \ell=1}^{m} \sum_{\alpha=1}^{n} \widetilde{R}_{i j k \ell} \varphi_{\alpha}^{i} \varphi_{0}^{j} \varphi \frac{k}{\alpha} \varphi_{0}^{\ell}
$$


Let $\partial y_{i}=\partial / \partial y^{i}$ be the local coordinate of $N$ and $\widetilde{R}(U, V, W, Z):=\langle\widetilde{R}(W, Z) V, U\rangle$. Thus

$$
\begin{aligned}
& \widetilde{R}_{i j k \ell} \varphi_{\alpha}^{i} \varphi_{\beta}^{j} \varphi \frac{k}{\alpha} \varphi \frac{\ell}{\beta} \\
= & \left\langle\widetilde{R}\left(\varphi_{\alpha}^{k} \partial y_{k}, \varphi \frac{\ell}{\beta} \partial y_{\ell}\right) \varphi_{\beta}^{j} \partial y_{j}, \varphi_{\alpha}^{i} \partial y_{i}\right\rangle=\widetilde{R}\left(X_{\alpha}, Y_{\beta}, \overline{X_{\alpha}}, \overline{Y_{\beta}}\right) \\
= & \widetilde{R}\left(\operatorname{Re}\left(X_{\alpha}\right), \operatorname{Re}\left(Y_{\beta}\right), \operatorname{Re}\left(X_{\alpha}\right), \operatorname{Re}\left(Y_{\beta}\right)\right)+\widetilde{R}\left(\operatorname{Re}\left(X_{\alpha}\right), \operatorname{Im}\left(Y_{\beta}\right), \operatorname{Re}\left(X_{\alpha}\right), \operatorname{Im}\left(Y_{\beta}\right)\right) \\
& +\widetilde{R}\left(\operatorname{Im}\left(X_{\alpha}\right), \operatorname{Re}\left(Y_{\beta}\right), \operatorname{Im}\left(X_{\alpha}\right), \operatorname{Re}\left(Y_{\beta}\right)\right)+\widetilde{R}\left(\operatorname{Im}\left(X_{\alpha}\right), \operatorname{Im}\left(Y_{\beta}\right), \operatorname{Im}\left(X_{\alpha}\right), \operatorname{Im}\left(Y_{\beta}\right)\right) \\
& -2 \widetilde{R}\left(\operatorname{Re}\left(X_{\alpha}\right), \operatorname{Re}\left(Y_{\beta}\right), \operatorname{Im}\left(X_{\alpha}\right), \operatorname{Im}\left(Y_{\beta}\right)\right)+2 \widetilde{R}\left(\operatorname{Re}\left(X_{\alpha}\right), \operatorname{Im}\left(Y_{\beta}\right), \operatorname{Im}\left(X_{\alpha}\right), \operatorname{Re}\left(Y_{\beta}\right)\right),
\end{aligned}
$$

where $X_{\alpha}=\varphi_{\alpha}^{i} \partial y_{i}, Y_{\beta}=\varphi_{\beta}^{j} \partial y_{j}$. Similarly, we have

$$
\begin{aligned}
& \widetilde{R}_{i j k \ell} \varphi_{\alpha}^{i} \varphi_{\bar{\beta}}^{j} \varphi_{\frac{k}{\alpha}} \varphi_{\beta}^{\ell} \\
= & \left\langle\widetilde{R}\left(\varphi_{\alpha}^{k} \partial y_{k}, \varphi_{\beta}^{\ell} \partial y_{\ell}\right) \varphi_{\bar{\beta}}^{j} \partial y_{j}, \varphi_{\alpha}^{i} \partial y_{i}\right\rangle=\widetilde{R}\left(X_{\alpha}, \overline{Y_{\beta}}, \overline{X_{\alpha}}, Y_{\beta}\right) \\
= & \widetilde{R}\left(\operatorname{Re}\left(X_{\alpha}\right), \operatorname{Re}\left(Y_{\beta}\right), \operatorname{Re}\left(X_{\alpha}\right), \operatorname{Re}\left(Y_{\beta}\right)\right)+\widetilde{R}\left(\operatorname{Re}\left(X_{\alpha}\right), \operatorname{Im}\left(Y_{\beta}\right), \operatorname{Re}\left(X_{\alpha}\right), \operatorname{Im}\left(Y_{\beta}\right)\right) \\
& +\widetilde{R}\left(\operatorname{Im}\left(X_{\alpha}\right), \operatorname{Re}\left(Y_{\beta}\right), \operatorname{Im}\left(X_{\alpha}\right), \operatorname{Re}\left(Y_{\beta}\right)\right)+\widetilde{R}\left(\operatorname{Im}\left(X_{\alpha}\right), \operatorname{Im}\left(Y_{\beta}\right), \operatorname{Im}\left(X_{\alpha}\right), \operatorname{Im}\left(Y_{\beta}\right)\right) \\
& +2 \widetilde{R}\left(\operatorname{Re}\left(X_{\alpha}\right), \operatorname{Re}\left(Y_{\beta}\right), \operatorname{Im}\left(X_{\alpha}\right), \operatorname{Im}\left(Y_{\beta}\right)\right)-2 \widetilde{R}\left(\operatorname{Re}\left(X_{\alpha}\right), \operatorname{Im}\left(Y_{\beta}\right), \operatorname{Im}\left(X_{\alpha}\right), \operatorname{Re}\left(Y_{\beta}\right)\right)
\end{aligned}
$$

and so

$$
\begin{aligned}
& \widetilde{R}=\widetilde{R}_{i j k \ell} \varphi_{\alpha}^{i} \varphi_{\beta}^{j} \varphi \frac{k}{\alpha} \varphi \frac{\ell}{\beta}+\widetilde{R}_{i j k \ell} \varphi_{\alpha}^{i} \varphi_{\frac{j}{\beta}}^{j} \varphi_{\alpha}^{k} \varphi_{\beta}^{\ell} \\
= & 2 \widetilde{R}\left(\operatorname{Re}\left(X_{\alpha}\right), \operatorname{Re}\left(Y_{\beta}\right), \operatorname{Re}\left(X_{\alpha}\right), \operatorname{Re}\left(Y_{\beta}\right)\right)+2 \widetilde{R}\left(\operatorname{Re}\left(X_{\alpha}\right), \operatorname{Im}\left(Y_{\beta}\right), \operatorname{Re}\left(X_{\alpha}\right), \operatorname{Im}\left(Y_{\beta}\right)\right) \\
& +2 \widetilde{R}\left(\operatorname{Im}\left(X_{\alpha}\right), \operatorname{Re}\left(Y_{\beta}\right), \operatorname{Im}\left(X_{\alpha}\right), \operatorname{Re}\left(Y_{\beta}\right)\right)+2 \widetilde{R}\left(\operatorname{Im}\left(X_{\alpha}\right), \operatorname{Im}\left(Y_{\beta}\right), \operatorname{Im}\left(X_{\alpha}\right), \operatorname{Im}\left(Y_{\beta}\right)\right) .
\end{aligned}
$$

Furthermore, similar computations show that

$$
\widetilde{R}^{0}=\widetilde{R}\left(\operatorname{Re}\left(X_{\alpha}\right), Z, \operatorname{Re}\left(X_{\alpha}\right), Z\right)+\widetilde{R}\left(\operatorname{Im}\left(X_{\alpha}\right), Z, \operatorname{Im}\left(X_{\alpha}\right), Z\right),
$$

where $X_{\alpha}=\varphi_{\alpha}^{i} \partial y_{i}$ and $Z=\varphi_{0}^{i} \partial y_{i}$. Therefore, if the sectional curvature $\widetilde{K}^{N}$ of $N$ is nonpositive, we see that

$$
\widetilde{R} \leq 0 \quad \text { and } \quad \widetilde{R}^{0} \leq 0 .
$$

Now by taking $\varepsilon=2$ in (3.6) we obtain

$$
\begin{aligned}
\left(\frac{\partial}{\partial t}-\Delta_{b}\right) \widehat{e}(\varphi) & \leq \sum_{k=1}^{m}\left[(2 R i c-(n-2) \text { Tor })\left(\left(\nabla_{b} \varphi^{k}\right)_{C},\left(\nabla_{b} \varphi^{k}\right)_{C}\right)+\frac{1}{2}\left|\nabla_{b} \varphi^{k}\right|^{2}\right] \\
& \leq C \sum_{k=1}^{m}\left|\nabla_{b} \varphi^{k}\right|^{2} \leq C \widehat{e}(\varphi) .
\end{aligned}
$$

Here $C$ is a positive constant depends on the pseudohermitian Ricci tensor and torsion of $(M, J, \theta)$.

Before we go further, let's recall Moser's Harnack inequality ([M]). Let

$$
\mathcal{L}=\frac{\partial}{\partial t}-\Delta_{b}
$$


be the heat operator on $\left(M^{2 n+1}, J, \theta\right)$. For $z_{0}=\left(x_{0}, t_{0}\right) \in M \times(0, \infty)$, let

$$
0<\delta<\operatorname{diam}(M), \quad 0<\tau<t_{0}
$$

and let $R\left(z_{0}, \delta, \tau\right)$ be the cylinder

$$
R\left(z_{0}, \delta, \tau\right)=\left\{(x, t) \in M \times[0, \infty):\left|x-x_{0}\right|<\delta, t_{0}-\tau<t<t_{0}\right\} .
$$

We have the following

LEMMA 3.3. Let u be a positive smooth solution of

$$
\mathcal{L} u \leq 0
$$

in $R\left(z_{0}, \delta, \tau\right)$. Then we have

$$
u\left(z_{0}\right) \leq C \int_{R\left(z_{0}, \delta, \tau\right)} u(x, t) d \mu d t,
$$

where $C>0$ is a constant depends only on $n, \delta$ and $\tau$.

To prove our main theorem, we need one more lemma.

Lemma 3.4. Let $\left(M^{2 n+1}, J, \theta\right)$ be a closed pseudohermitian manifold and $\left(N^{m}, g\right)$ be a Riemannian manifold with nonpositive sectional curvature $\widetilde{K}^{N}$. For any $0<T \leq$ $\infty$, if $\varphi \in C^{\infty}(M \times[0, T) ; N)$ solves (1.2), then

$$
\widehat{E}(\varphi(t)):=E(\varphi(t))+E^{0}(\varphi(t))
$$

is decreasing in $t$. Here $E^{0}(\varphi(t))$ is given by

$$
E^{0}(\varphi(t)):=\int_{M} e_{0}(\varphi) d \mu=\int_{M} g_{i j} \varphi_{0}^{i} \varphi_{0}^{j} d \mu .
$$

REMARK 3.1. The energy $E^{0}(\varphi(t))$ is decreasing in $t$ under the flow only in case of $\left(N^{m}, g\right)$ with nonpositive sectional curvature $\widetilde{K}^{N}$.

Proof. From (3.5) and (3.7) one has

$$
\begin{aligned}
\frac{d}{d t} E^{0}(\varphi(t)) & =\int_{M} \frac{\partial}{\partial t}\left(e_{0}(\varphi)\right) d \mu \\
& =\int_{M}\left[4 \widetilde{R}^{0}-2 \sum_{k=1}^{m}\left|\nabla_{b} \varphi_{0}^{k}\right|^{2}\right] d \mu \leq 0,
\end{aligned}
$$

provided the sectional curvature $\widetilde{K}^{N}$ of $N$ is nonpositive. This says that $E^{0}(\varphi(t))$ is decreasing in $t$. Since $E(\varphi(t))$ is also decreasing in $t$ (see Lemma 3.1), we then conclude that $\widehat{E}(\varphi(t))$ is decreasing in $t$.

Proof of Theorem 1.3. We first show that $\left|\nabla_{b} \varphi\right|$ is uniformly bounded. In fact, we will show that $\widehat{e}(\varphi)$ is uniformly bounded. Let

$$
F(x, t):=e^{-C t} \widehat{e}(\varphi(x, t)), \quad(x, t) \in M \times[0, T) .
$$


Here $C>0$ is a constant satisfying

$$
\left(\frac{\partial}{\partial t}-\Delta_{b}\right) \widehat{e}(\varphi) \leq C \widehat{e}(\varphi) .
$$

It is easy to check that

$$
\left(\frac{\partial}{\partial t}-\Delta_{b}\right) F(x, t)=e^{-C t}\left[\left(\frac{\partial}{\partial t}-\Delta_{b}\right) \widehat{e}(\varphi)-C \widehat{e}(\varphi)\right] \leq 0
$$

and then for any $z_{0}=\left(x_{0}, t_{0}\right) \in M \times[0, T)$ we have by Lemma 3.3 , that

$$
\begin{aligned}
\widehat{e}\left(\varphi\left(z_{0}\right)\right) & \leq C_{1} e^{C t_{0}} \int_{R\left(z_{0}, \delta, 1\right)} F(x, s) d \mu d s \\
& =C_{1} \int_{t_{0}-1}^{t_{0}} \int_{B_{\delta}\left(x_{0}\right)} e^{C\left(t_{0}-s\right)} \widehat{e}(\varphi(x, s)) d \mu d s \\
& \leq C_{1} \int_{t_{0}-1}^{t_{0}} \int_{B_{\delta}\left(x_{0}\right)} \widehat{e}(\varphi(x, s)) d \mu d s \\
& \leq C_{1} \int_{t_{0}-1}^{t_{0}} \widehat{E}(\varphi(s)) d s \leq C_{1} \widehat{E}(f),
\end{aligned}
$$

since $\widehat{E}(\varphi(t))$ is decreasing in $t$ by Lemma 3.4. This shows that $\left|\nabla_{b} \varphi\right|$ is uniformly bounded and we conclude that $\varphi \in C^{\infty}(M \times[0, \infty) ; N)$ by the higher order regularity of parabolic equations (see $[\mathrm{F}]$ and $[\mathrm{CCC}]$ ).

Since from Lemma 3.1,

$$
\frac{1}{2} \int_{0}^{t} \int_{M}\left|\partial_{s} \varphi\right|^{2} d \mu d s \leq E(f)<+\infty, \quad \forall t
$$

we see that

$$
\lim _{t \uparrow \infty} \int_{t-2}^{t} \int_{M}\left|\partial_{s} \varphi\right|^{2} d \mu d s=0 .
$$

By a direct computation one has

$$
\begin{aligned}
& \left(\frac{\partial}{\partial t}-\Delta_{b}\right)\left|\partial_{t} \varphi\right|^{2} \\
= & -2 \sum_{k=1}^{m}\left|\nabla_{b}\left(\frac{\partial \varphi^{k}}{\partial t}\right)\right|^{2}+4 \sum_{i, j, k, \ell=1}^{m} \sum_{\alpha=1}^{n} \widetilde{R}_{i j k \ell} \varphi_{\alpha}^{i} \frac{\partial \varphi^{j}}{\partial t} \varphi \frac{k}{\alpha} \frac{\partial \varphi^{\ell}}{\partial t} \\
= & -2 \sum_{k=1}^{m}\left|\nabla_{b}\left(\frac{\partial \varphi^{k}}{\partial t}\right)\right|^{2} \\
& +4 \sum_{\alpha=1}^{n}\left[\widetilde{R}\left(\operatorname{Re}\left(X_{\alpha}\right), W, \operatorname{Re}\left(X_{\alpha}\right), W\right)+\widetilde{R}\left(\operatorname{Im}\left(X_{\alpha}\right), W, \operatorname{Im}\left(X_{\alpha}\right), W\right)\right],
\end{aligned}
$$

where $X_{\alpha}=\varphi_{\alpha}^{i} \partial y_{i}$ and $W=\frac{\partial \varphi^{i}}{\partial t} \partial y_{i}$ with $\partial y_{i}=\partial / \partial y^{i}$ the local coordinates of $N$. Thus, the sectional curvature $\widetilde{K}^{N}$ of $N$ is nonpositive implies that

$$
\left(\frac{\partial}{\partial t}-\Delta_{b}\right)\left|\partial_{t} \varphi\right|^{2} \leq 0
$$


and so by Lemma 3.3 we see that,

$$
\left\|\partial_{t} \varphi\right\|_{C^{0}(M \times[t-1, t])} \leq C\left\|\partial_{t} \varphi\right\|_{L^{2}(M \times[t-2, t])} \rightarrow 0
$$

as $t \rightarrow \infty$. Therefore, we may choose a sequence $\left\{t_{\ell}\right\}_{\ell}$ with $t_{\ell} \uparrow \infty$ as $\ell \rightarrow \infty$ such that $\varphi_{t}\left(\cdot, t_{\ell}\right) \rightarrow 0$ in $C^{0}(M)$ and $\varphi\left(\cdot, t_{\ell}\right) \rightarrow \varphi_{\infty}$ in $C^{2}(M ; N)$. It is easy to see that the limit map $\varphi_{\infty}$ satisfies the Euler-Lagrange equations

$$
\begin{aligned}
\Delta_{b}\left(\varphi_{\infty}\right)^{k}+2 \widetilde{\Gamma}_{i j}^{k}\left(\varphi_{\infty}\right)_{\alpha}^{i}\left(\varphi_{\infty}\right) \frac{j}{\alpha} & =\lim _{\ell \rightarrow \infty}\left[\Delta_{b} \varphi^{k}\left(\cdot, t_{\ell}\right)+2 \widetilde{\Gamma}_{i j}^{k} \varphi_{\alpha}^{i}\left(\cdot, t_{\ell}\right) \varphi_{\bar{\alpha}}^{j}\left(\cdot, t_{\ell}\right)\right] \\
& =\lim _{\ell \rightarrow \infty} \varphi_{t}\left(\cdot, t_{\ell}\right)=0
\end{aligned}
$$

and we see that $\varphi \in C^{\infty}(M ; N)$ by the general theory of subelliptic equations. Since $\varphi_{\infty} \in C^{\infty}(M ; N)$ satisfies the Euler-Lagrange equations (3.8), we then conclude by Lemma 2.2 that $\varphi_{\infty}$ is a pseudoharmonic map.

\section{REFERENCES}

[BDU] E. Barletta, S. Dragomir and H. Urakawa, Pseudoharmonic Maps from Nondegenerate CR Manifolds to Riemannian Manifolds, IUMJ, 50 (2001), pp. 719-746.

[CCC] S.-C., Chang, J.-H. Cheng, And H.-L. Chiu, The Fourth-order Q-curvature flow on a CR 3-manifold, Indiana Univ. Math. J., 56:4 (2007), pp. 1793-1826.

[CC1] S.-C. Chang AND H.-L. Chiu, Nonnegativity of CR Paneitz operator and its Application to the CR Obata's Theorem in a Pseudohermitian $(2 n+1)$-Manifold, JGA, 19 (2009), pp. 261-287.

[CC2] S.-C. Chang And H.-L. Chiu, On the CR Analogue of Obata's Theorem in a Pseudohermitian 3-Manifold, Math. Ann., 345 (2009), pp. 33-51.

[DT] S. Dragomir and G. Tomassini, Differential Geometry and Analysis on CR Manifolds, Progress in Mathematics, 246. Birkhauser Boston, Inc., Boston, MA, 2006. xvi+487 pp.

[ES] J. Eells AND J. H. SAmpson, Harmonic Mappings of Riemannian Manifolds, Amer. J. Math., 86 (1964), pp. 109-160.

[F] G. B. Folland, Subelliptic estimates and function spaces on nilpotent Lie groups, ARKIV FÖR MATEMATIK, 13:1-2 (1975), pp. 161-207.

[FS] G. B. Folland And E. M. SteIn, Estimates for the $\bar{\partial}_{b}$ complex and analysis on the Heisenberg group, Comm. Pure Appl. Math., 27 (1974), pp. 429-522.

[GG] A. R. Gover and C. R. Graham, CR Invariant Powers of the Sub-Laplacian, J. Reine Angew. Math., 583 (2005), pp. 1-27.

[GL] C. R. Graham And J. M. LeE, Smooth Solutions of Degenerate Laplacians on Strictly Pseudoconvex Domains, Duke Math. J., 57 (1988), pp. 697-720.

[H] K. HiRACHI, Scalar Pseudo-hermitian Invariants and the Szegö Kernel on 3-dimensional CR Manifolds, Lecture Notes in Pure and Appl. Math., 143 (1992), pp. 67-76, Dekker.

[Ho] L. Hörmander, Hypoelliptic differential operators, Annales, de l'institut Fourier, tome 11 (1961), pp. 477-492.

[JX] J. Jost And C.-J. Xu, Subelliptic Harmonic Maps, Trans. of AMS, 350:11 (1998), pp. 46334649 .

[Lie] G. M. Lieberman, Second order parabolic differential equations, World Scientific Publishing Co., Inc., River Edge, NJ, 1996.

[L1] J. M. LEe, Pseudo-Einstein Structure on CR Manifolds, Amer. J. Math., 110 (1988), pp. $157-178$.

[L2] J. M. LEE, The Fefferman Metric and Pseudohermitian Invariants, Trans. A.M.S., 296 (1986), pp. 411-429.

[LW] F. Lin And C.-Y. Wang, The Analysis of Harmonic Maps and Their Heat Flows, World Scientific, 2008.

[M] Jürgen Moser, A Harnack Inequality for Parabolic Differential Equations, Communications on pure and applied mathematics, 17 (1964), pp. 101-134.

[P] S. Paneitz, A Quartic Conformally Covariant Differential Operator for Arbitrary PseudoRiemannian Manifolds, preprint, 1983.

[Pe] R. Petit, Harmonic maps and strictly pseudoconvex CR manifolds, Comm. Anal. Geom., 10:3 (2002), pp. 575-610. 
[SS] J. Shatah and M. Struwe, Geometric wave equations, Courant Lecture Notes in Mathematics, 2. New York University, Courant Institute of Mathematical Sciences, New York; American Mathematical Society, Providence, RI, 1998.

[SY] R. Schoen and S.-T. Yau, Lectures on Differential Geometry, International Press, 1994.

[W] J.-P. WANG, private communication, 2005. 